\title{
Efficient Calibration of the Non-Conservative Force Data from the Space-Borne Accelerometers of the Twin GRACE Satellites
}

\author{
By Wei ZHENG,, ${ }^{1), 2)}$ Houtse Hsu, ${ }^{1)}$ Min ZHONG ${ }^{1)}$ and Meijuan YUN $^{3)}$ \\ ${ }^{1)}$ Key Laboratory of Dynamic Geodesy, Institute of Geodesy and Geophysics, Chinese Academy of Sciences, Wuhan, China \\ ${ }^{2)}$ Disaster Prevention Research Institute, Kyoto University, Uji, Japan \\ ${ }^{3)}$ Department of Applied Physics, Wuhan University of Science and Technology, Wuhan, China
}

(Received May 28th, 2010)

\begin{abstract}
Different from calibrations previously conducted using the dynamic method, the GRACE-Level-1B non-conservative force data from the space-borne accelerometers between 1 January to 31 December, 2009, released by the Jet Propulsion Laboratory in the United States of America, is for the first time ever effectively calibrated using the priori Earth gravitational field model based on an improved energy conservation principle. The results are shown as follows: 1) The non-conservative force data from the space-borne accelerometers and attitude data from the star camera assembly are basically the same as the anticipated accuracies; 2) the systematic error of the raw non-conservative force data leads to a disturbed geopotential error linear drift of about $0.4 \mathrm{~m}^{2} / \mathrm{s}^{2}$ per day, however, the error is only $0.01 \mathrm{~m}^{2} / \mathrm{s}^{2}$ using the calibrated non-conservative force data, and a reasonable physical explanation is provided; 3 ) the advantages and disadvantages of calibrating non-conservative force data using the energy conservation principle and dynamic method are compared in detail; and 4) because the signal of the Earth's gravitational field is sensitive to the systematic error of non-conservative force data from the space-borne accelerometers, the precise calibration of non-conservative force data is a key factor for the highly accurate recovery of the Earth gravitational field with high spatial resolution.
\end{abstract}

Key Words: Twin GRACE Satellites, Space-Borne Accelerometer, Non-Conservative Force, Calibration, Energy Conservation Principle

\section{Introduction}

The 21 st century is a new era in which human beings are upgrading their cognitive capabilities to a digital-based Earth using satellite-to-satellite tracking in the high-low mode (SST-HL), satellite-to-satellite tracking in the lowlow mode (SST-LL) and satellite gravity gradiometry (SGG). Since the successful launching of the first Sputnik1 satellite on October 4, 1957, many scholars have carried out all kinds of wide-ranging and extensive studies on satellite gravity measurement. ${ }^{1-14)}$ The Earth's static and timevarying gravitational field can reflect the spatial distribution, movement and alteration of materials on and inside the Earth, and can dominate the undulation and changes of the geoid. Accordingly, investigating the detailed configuration and time-variable characteristics of the Earth's gravitational field not only is required for satellite geodesy, space science, astronautics, geophysics, seismology, oceanography, and so on, but also provides important information for resource exploration, environmental protection and disaster monitoring. Two Gravity Recovery and Climate Experiment satellites (i.e., twin GRACE satellites) were launched on March 17, 2002. They are designed to fly in an almost circular orbit (orbital eccentricity of 0.004), near the polar axis (orbital inclination of $89^{\circ}$ ) and at a Low-Earth-Orbit altitude (orbital altitude of $500-300 \mathrm{~km}$ ) during a 5-10 yr flight mission. In order to determine the effects of non-conservative force,

(C) 2011 The Japan Society for Aeronautical and Space Sciences the twin GRACE satellites each carry an electrostatically suspended SuperSTAR accelerometer located at the center of mass of the satellite. The accelerometer has an anticipated resolution of $1 \times 10^{-10} \mathrm{~m} / \mathrm{s}^{2}$ over the frequency band from $10^{-4}$ to $10^{-1} \mathrm{~Hz}$.

The GRACE mission has been successfully achieved owing to the persevering exploration by many international research institutions for over $30 \mathrm{yr}$. Understanding that many opportunities and challenges await us, the authors suggest to implement a next-generation satellite gravity measurement program as soon as possible in the near future by utilizing the successful experiences accumulated internationally over the many years as a reference. At present, many scholars are closely following the development trends in international satellite gravity measurement missions, and are actively participating in the analysis of requirements for optimal selection such as tracking modes, key payloads, computing methods and data processing. Based on the above activities, this study includes the following: 1) The basic parameters of the GRACE-Level-1B non-conservative force data from the space-borne accelerometers provided by the Jet Propulsion Laboratory (JPL) and the corresponding relationship between accelerometer frame (AF) and satellite frame (SF) are introduced; 2) the physical and mathematical models including calibration of the non-conservative force data, use of attitude data and the coordinate transformation of calibrated non-conservative force data are presented; and 3) different from previous calibration using the dynamic 


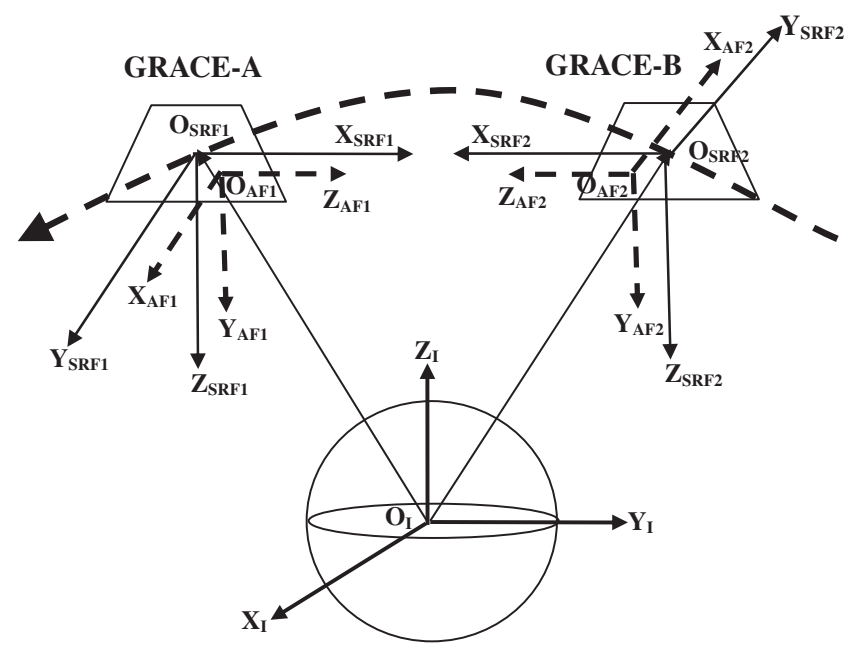

Fig. 1. Science reference frame (SRF) and accelerometer frame (AF).

method, ${ }^{15,16)}$ for the first time ever, we effectively calibrated the non-conservative force data from the space-borne accelerometers using the priori Earth gravitational field model based on an improved energy conservation principle. This work will provide the theoretical foundation and calculational guarantee for the efficient calibration of non-conservative force data from the space-borne accelerometers used in the next-generation satellite gravity measurement missions.

\section{Methods}

As illustrated in Fig. 1, the GRACE-Level-1B nonconservative force data from the space-borne accelerometers, released by the JPL, is located in the Science Reference Frame (SRF), and is presented orderly using a data file with 1-s sample intervals per day (2009-01-01 00:00:00.0023:59:59.00). In the SF, the origin $O_{\mathrm{SF}}$ is located at the centre of satellite mass, the positive direction of $X_{\mathrm{SF}}$ is from the origin to the target location of the phase center of the $\mathrm{K} / \mathrm{Ka}$ band horn, and the positive directions of $X_{\mathrm{SF} 1}$ and $X_{\mathrm{SF} 2}$ are collinear reversely. The positive direction of $Z_{\mathrm{SF}}$ towards the satellite radiator is normal to $X_{\mathrm{SF}}$, and $Y_{\mathrm{SF}}$ forms a right-handed triad with $X_{\mathrm{SF}}$ and $Z_{\mathrm{SF}}$. In the AF, the origin $O_{\mathrm{AF}}$ is located at the centre of accelerometer mass, the three-axis definition being $X_{\mathrm{AF}} \cong+Y_{\mathrm{SF}}, Y_{\mathrm{AF}} \cong+Z_{\mathrm{SF}}$ and $Z_{\mathrm{AF}} \cong+X_{\mathrm{SF}}$. In the SRF, the origin $O_{\mathrm{SRF}}$ is located at the centre of satellite mass, and the triaxial definition is $X_{\mathrm{SRF}} \equiv+Z_{\mathrm{AF}}, Y_{\mathrm{SRF}} \equiv+X_{\mathrm{AF}}$ and $Z_{\mathrm{SRF}} \equiv+Y_{\mathrm{AF}}$.

Since the non-conservative force data from the GRACE space-borne accelerometers were released, many international research organizations such as the American JPL and the German GeoForschungsZentrum Potsdam (GFZ) have conducted related studies about processing the nonconservative force data. The results show that there is the systematic error in the non-conservative force data, and the accuracy of the Earth's gravitational field will be reduced to a greater degree if the systematic error is not revised effectively. Before calibration with scale and bias factors, the non-conservative force data from the spaceborne accelerometers needs to have the coordinate transformed from SRF to AF:

$$
\left[\begin{array}{l}
a_{x} \\
a_{y} \\
a_{z}
\end{array}\right]_{\mathrm{AF}}=B\left[\begin{array}{c}
a_{x}^{0} \\
a_{y}^{0} \\
a_{z}^{0}
\end{array}\right]_{\mathrm{SRF}}
$$

where $\left[\begin{array}{lll}a_{x} & a_{y} & a_{z}\end{array}\right]_{\mathrm{AF}}^{\mathrm{T}}$ and $\left[\begin{array}{lll}a_{x}^{0} & a_{y}^{0} & a_{z}^{0}\end{array}\right]_{\mathrm{SRF}}^{\mathrm{T}}$ respectively denote the non-conservative force data in the AF and $\mathrm{SRF}$, and $\boldsymbol{B}$ shows the transformation matrix between SRF and AF:

$$
\boldsymbol{B}=\left[\begin{array}{lll}
0 & 1 & 0 \\
0 & 0 & 1 \\
1 & 0 & 0
\end{array}\right]
$$

The calibration of non-conservative force data is expressed as:

$$
f_{i j}^{0}=b_{i j}+k_{i j} a_{i j}
$$

where subscript $i$ represents GRACE-A/B, $i=1,2$, and subscript $j$ denotes the $x, y$ and $z$ axes, $j=1,2,3 . a_{i j}$ and $f_{i j}^{0}$ show raw and calibrated non-conservative force data, and $k_{i j}$ and $b_{i j}$ are scale and bias factors, respectively.

Due to the fluctuation of systematic error from the spaceborne accelerometers over time, the scale factor provided by the JPL is only the average value of the total calibrated nonconservative force data, and the bias factor is presented using an approximation formula with a Modified Julian Date (MJD); therefore, the actual change in systematic error per day is not reflected accurately. When the Earth's gravitational field is recovered using the non-conservative force data within a special time range, the scale and bias factors must be recalibrated accurately.

The GRACE-Level-1B attitude data from the star camera assembly provided by the JPL is presented orderly using one data file each day (2009-01-01 00:00:00.00-23:59:59.00) at 5-s sample intervals. Different from Euler angles used in the traditional method, quaternion is used to define the threeaxis attitude of the GRACE space-borne accelerometers. The transformation matrix of quaternion from SRF to Earth-centered Inertial frame (ECI) is expressed as:

$$
C(q)=\left[\begin{array}{ccc}
q_{1}^{2}-q_{2}^{2}-q_{3}^{2}+q_{4}^{2} & 2\left(q_{1} q_{2}+q_{3} q_{4}\right) & 2\left(q_{1} q_{3}-q_{2} q_{4}\right) \\
2\left(q_{1} q_{2}-q_{3} q_{4}\right) & -q_{1}^{2}+q_{2}^{2}-q_{3}^{2}+q_{4}^{2} & 2\left(q_{2} q_{3}+q_{1} q_{4}\right) \\
2\left(q_{1} q_{3}+q_{2} q_{4}\right) & 2\left(q_{2} q_{3}-q_{1} q_{4}\right) & -q_{1}^{2}-q_{2}^{2}+q_{3}^{2}+q_{4}^{2}
\end{array}\right] .
$$


Because an energy observation equation is established in the ECI, all of the satellite observations need to be uniformly transformed into ECI. The coordinate of calibrated non-conservative force data is transformed from $\mathrm{AF}$ to ECI:

$$
\left[\begin{array}{c}
f_{x} \\
f_{y} \\
f_{z}
\end{array}\right]_{\mathrm{ECI}}=C(q) B^{\mathrm{T}}\left[\begin{array}{c}
f_{x}^{0} \\
f_{y}^{0} \\
f_{z}^{0}
\end{array}\right]_{\mathrm{AF}}
$$

where $\left[\begin{array}{lll}f_{x} & f_{y} & f_{z}\end{array}\right]_{\mathrm{ECI}}^{\mathrm{T}}$ and $\left[\begin{array}{lll}f_{x}^{0} & f_{y}^{0} & f_{z}^{0}\end{array}\right]_{\mathrm{AF}}^{\mathrm{T}}$ are the nonconservative force data in the ECI and AF, respectively.

Different from the energy observation equation with reference-disturbing geopotential established by Jekeli ${ }^{17}$ ) and $\mathrm{Han},{ }^{18)}$ we have developed the new energy observation equation without reference-disturbing geopotential. Under guaranteeing the accuracy of the Earth gravitational field recovery, the energy observation equation is simplified and the computing speed is enhanced. Calibrating the nonconservative force data using the priori Earth gravitational field model based on the improved energy conservation principle is one effective way to cancel systematic error. In the ECI, the observation equation for disturbing geopotential difference between the twin satellites is defined as:

$$
\boldsymbol{T}_{\mathrm{e} 12}=\boldsymbol{E}_{\mathrm{k} 12}-\boldsymbol{E}_{\mathrm{f} 12}+\boldsymbol{V}_{\omega 12}-\boldsymbol{V}_{\mathrm{T} 12}-\boldsymbol{V}_{012}-\boldsymbol{E}_{012}
$$

where $\boldsymbol{T}_{\mathrm{e} 12}$ represents the disturbing geopotential difference of the twin satellites on the left-hand side:

$$
\begin{aligned}
\boldsymbol{T}_{\mathrm{e} 12}\left(r_{1}, \theta_{1}, \lambda_{1}, r_{2}, \theta_{2}, \lambda_{2}\right) \\
=\frac{G M}{R_{\mathrm{e}}} \sum_{l=2}^{L} \sum_{m=-l}^{l}\left\{\left[\left(\frac{R_{\mathrm{e}}}{r_{2}}\right)^{l+1} \bar{Y}_{l m}\left(\theta_{2}, \lambda_{2}\right)\right.\right. \\
\left.\left.\quad-\left(\frac{R_{\mathrm{e}}}{r_{1}}\right)^{l+1} \bar{Y}_{l m}\left(\theta_{1}, \lambda_{1}\right)\right] \overline{\boldsymbol{C}}_{l m}\right\}
\end{aligned}
$$

where $\bar{Y}_{l, m}(\theta, \lambda)=\bar{P}_{l,|m|}(\cos \theta) Q_{m}(\lambda)$,

$$
Q_{m}(\lambda)=\left\{\begin{array}{ll}
\cos m \lambda & m \geq 0 \\
\sin |m| \lambda & m<0
\end{array} ;\right.
$$

GM indicates the product of the Earth's mass $M$ and gravitational constant $G ; R_{\mathrm{e}}$ expresses the Earth's mean radius; $r_{1}$ and $r_{2}$ are the geocentric radii of the twin satellites $r_{1(2)}=\sqrt{x_{1(2)}^{2}+y_{1(2)}^{2}+z_{1(2)}^{2}}, x_{1(2)}, y_{1(2)}$ and $z_{(2)}$ show the components of position vector $\boldsymbol{r}_{1(2)} ; \theta_{1}$ and $\theta_{2}$ are geocentric colatitudes; $\lambda_{1}$ and $\lambda_{2}$ are geocentric longitudes; $\bar{P}_{l m}(\cos \theta)$ represents the normalized Legendre polynomial with degree $l$ and order $m$; and $\overline{\boldsymbol{C}}_{l m}$ denotes the estimated geopotential coefficient. The first term on the right-hand side,

$$
\boldsymbol{E}_{\mathrm{k} 12}=\frac{1}{2}\left(\dot{\boldsymbol{r}}_{2}+\dot{\boldsymbol{r}}_{1}\right) \cdot\left\{\dot{\rho}_{12} \boldsymbol{e}_{12}+\left[\dot{\boldsymbol{r}}_{12}-\left(\dot{\boldsymbol{r}}_{12} \cdot \boldsymbol{e}_{12}\right) \boldsymbol{e}_{12}\right]\right\}
$$

is the kinetic energy difference of the twin satellites, where $\dot{\rho}_{12}$ denotes the inter-satellite range-rate measurement along the track from the K-band ranging system, $\dot{\boldsymbol{r}}_{1}$ and $\dot{\boldsymbol{r}}_{2}$ represent absolute velocity vectors, $\dot{\boldsymbol{r}}_{12}=\dot{\boldsymbol{r}}_{2}-\dot{\boldsymbol{r}}_{1}$ shows relative velocity vector between GRACE satellites, and $\boldsymbol{e}_{12}=$ $\boldsymbol{r}_{12} /\left|\boldsymbol{r}_{12}\right|$ indicates the unit vector identifying the direction to the second satellite from the first. The second term,

$$
\boldsymbol{E}_{\mathrm{f} 12}=\int\left(\dot{\boldsymbol{r}}_{2} \cdot \boldsymbol{f}_{2}-\dot{\boldsymbol{r}}_{1} \cdot \boldsymbol{f}_{1}\right) \mathrm{d} t
$$

is the dissipative energy difference acting on the twin satellites, where $\boldsymbol{f}_{1}$ and $\boldsymbol{f}_{2}$ express non-conservative forces. The third term, $\boldsymbol{V}_{\omega 12}=-\omega_{\mathrm{e}}\left(x_{12} \dot{y}_{2}-y_{2} \dot{x}_{12}-y_{12} \dot{x}_{1}+x_{1} \dot{y}_{12}\right)$, is the geopotential rotation difference of the twin satellites, where $\omega_{\mathrm{e}}$ denotes the Earth's angular rotation rate. The fourth term, $\boldsymbol{V}_{\mathrm{T} 12}$, is equal to third-body perturbation potential difference, such as the Sun, Moon, Earth's solid tides and general principle of relativity. The fifth term, $\boldsymbol{V}_{012}=G M / r_{2}-G M / r_{1}$, is the geocentric potential difference. The last term, $\boldsymbol{E}_{012}$, is the energy integral constant difference of the twin satellites system, derived from the initial position and velocity.

\section{Results}

In this study, the main ideas for calibrating the nonconservative force data from the space-borne accelerometers are as follows. First, the disturbing geopotential difference is calculated using the priori Earth gravitational field model. Second, the scale and bias factors of the non-conservative force data in the AF are fitted by the least-squares method based on the improved energy conservation principle. Equation (6) is modified as:

$$
\begin{aligned}
& \int\left(\dot{\boldsymbol{r}}_{2} \cdot \boldsymbol{f}_{2}-\dot{\boldsymbol{r}}_{1} \cdot \boldsymbol{f}_{1}\right) \mathrm{d} t \\
& \quad=\boldsymbol{E}_{\mathrm{k} 12}+\boldsymbol{V}_{\omega 12}-\boldsymbol{V}_{\mathrm{T} 12}-\boldsymbol{V}_{012}-\boldsymbol{T}_{\mathrm{e} 12}-\boldsymbol{E}_{012} .
\end{aligned}
$$

Assuming the right-hand side of Eq. (6) to be $\boldsymbol{E}_{12}=\boldsymbol{E}_{\mathrm{k} 12}+\boldsymbol{V}_{\omega 12}-\boldsymbol{V}_{\mathrm{T} 12}-\boldsymbol{V}_{012}-\boldsymbol{T}_{\mathrm{e} 12}-\boldsymbol{E}_{012}$, and $n$ observations with corresponding times $t_{1}, t_{2}, \cdots, t_{n}$, the discrete energy observation equation is expressed as:

$$
\begin{aligned}
& \dot{\boldsymbol{r}}_{2}\left(t_{1}\right) \cdot \boldsymbol{f}_{2}\left(t_{1}\right)-\dot{\boldsymbol{r}}_{1}\left(t_{1}\right) \cdot \boldsymbol{f}_{1}\left(t_{1}\right)=\boldsymbol{E}_{12}\left(t_{1}\right) / \Delta t \\
& {\left[\dot{\boldsymbol{r}}_{2}\left(t_{1}\right) \cdot \boldsymbol{f}_{2}\left(t_{1}\right)-\dot{\boldsymbol{r}}_{1}\left(t_{1}\right) \cdot \boldsymbol{f}_{1}\left(t_{1}\right)\right]+\left[\dot{\boldsymbol{r}}_{2}\left(t_{2}\right) \cdot \boldsymbol{f}_{2}\left(t_{2}\right)-\dot{\boldsymbol{r}}_{1}\left(t_{2}\right) \cdot \boldsymbol{f}_{1}\left(t_{2}\right)\right]=\boldsymbol{E}_{12}\left(t_{2}\right) / \Delta t} \\
& \quad \ldots \\
& {\left[\dot{\boldsymbol{r}}_{2}\left(t_{1}\right) \cdot \boldsymbol{f}_{2}\left(t_{1}\right)-\dot{\boldsymbol{r}}_{1}\left(t_{1}\right) \cdot \boldsymbol{f}_{1}\left(t_{1}\right)\right]+\cdots+\left[\dot{\boldsymbol{r}}_{2}\left(t_{n}\right) \cdot \boldsymbol{f}_{2}\left(t_{n}\right)-\dot{\boldsymbol{r}}_{1}\left(t_{n}\right) \cdot \boldsymbol{f}_{1}\left(t_{n}\right)\right]=\boldsymbol{E}_{12}\left(t_{n}\right) / \Delta t .}
\end{aligned}
$$

Based on Eq. (9), the difference between the adjacent epochs is represented as: 
Table 1. Scale and bias factors for calibrating the non-conservative force data from the space-borne accelerometers.

\begin{tabular}{|c|c|c|c|c|c|c|c|}
\hline \multirow{2}{*}{\multicolumn{2}{|c|}{ Parameters }} & \multicolumn{2}{|c|}{ EGM2008 } & \multicolumn{2}{|c|}{ GGM02S } & \multicolumn{2}{|c|}{ EIGEN-5C } \\
\hline & & GRACE-A & GRACE-B & GRACE-A & GRACE-B & GRACE-A & GRACE-B \\
\hline \multirow{3}{*}{ Scale factor } & $X_{\mathrm{AF}}$ & $0.980 \pm 0.018$ & $0.960 \pm 0.016$ & $0.976 \pm 0.025$ & $0.955 \pm 0.019$ & $0.982 \pm 0.021$ & $0.961 \pm 0.018$ \\
\hline & $Y_{\mathrm{AF}}$ & $0.930 \pm 0.015$ & $0.920 \pm 0.012$ & $0.928 \pm 0.020$ & $0.916 \pm 0.018$ & $0.935 \pm 0.017$ & $0.922 \pm 0.014$ \\
\hline & $Z_{\mathrm{AF}}$ & $0.969 \pm 0.002$ & $0.955 \pm 0.002$ & $0.963 \pm 0.005$ & $0.951 \pm 0.004$ & $0.971 \pm 0.003$ & $0.959 \pm 0.002$ \\
\hline \multirow{3}{*}{$\begin{array}{l}\text { Bias factor } \\
\left(10^{-6} \mathrm{~m} / \mathrm{s}^{2}\right)\end{array}$} & $X_{\mathrm{AF}}$ & $30.960 \pm 0.015$ & $6.878 \pm 0.012$ & $30.955 \pm 0.019$ & $6.871 \pm 0.018$ & $30.963 \pm 0.017$ & $6.880 \pm 0.015$ \\
\hline & $Y_{\mathrm{AF}}$ & $-0.501 \pm 0.010$ & $-0.365 \pm 0.009$ & $-0.497 \pm 0.018$ & $-0.360 \pm 0.013$ & $-0.508 \pm 0.013$ & $-0.369 \pm 0.010$ \\
\hline & $Z_{\mathrm{AF}}$ & $-1.021 \pm 0.008$ & $-0.566 \pm 0.006$ & $-1.016 \pm 0.011$ & $-0.561 \pm 0.009$ & $-1.025 \pm 0.009$ & $-0.568 \pm 0.008$ \\
\hline
\end{tabular}

$$
\begin{gathered}
\dot{\boldsymbol{r}}_{2}\left(t_{1}\right) \cdot \boldsymbol{f}_{2}\left(t_{1}\right)-\dot{\boldsymbol{r}}_{1}\left(t_{1}\right) \cdot \boldsymbol{f}_{1}\left(t_{1}\right)=\tilde{\boldsymbol{E}}_{12}\left(t_{1}\right) \\
\dot{\boldsymbol{r}}_{2}\left(t_{2}\right) \cdot \boldsymbol{f}_{2}\left(t_{2}\right)-\dot{\boldsymbol{r}}_{1}\left(t_{2}\right) \cdot \boldsymbol{f}_{1}\left(t_{2}\right)=\tilde{\boldsymbol{E}}_{12}\left(t_{2}\right) \\
\cdots \\
\cdots \\
\dot{\boldsymbol{r}}_{2}\left(t_{n}\right) \cdot \boldsymbol{f}_{2}\left(t_{n}\right)-\dot{\boldsymbol{r}}_{1}\left(t_{n}\right) \cdot \boldsymbol{f}_{1}\left(t_{n}\right)=\tilde{\boldsymbol{E}}_{12}\left(t_{n}\right)
\end{gathered}
$$

where

$$
\tilde{\boldsymbol{E}}_{12}\left(t_{k}\right)=\frac{\boldsymbol{E}_{12}\left(t_{k}\right)-\boldsymbol{E}_{12}\left(t_{k-1}\right)}{\Delta t} \quad(k=2,3, \cdots, n) .
$$

The advantages of Eq. (10) are that the energy integral constant is cancelled through the difference of the adjacent epochs and the calculating process is simplified to a greater degree. As shown in Table 1, the non-conservative force data from the space-borne accelerometers were calibrated using the EGM2008 priori Earth gravitational field model based on the improved energy conservation principle once a day, and the scale and bias factors suitable for the time range from 2009-01-01 00:00:00.00 to 12-31 24:00:00.00 are fitted in the AF. As displayed in Fig. 2, the error of disturbing geopotential in Eq. (6) represents a linear drift of $0.4 \mathrm{~m}^{2} / \mathrm{s}^{2}$ per day using the raw non-conservative force data, and an error of $0.01 \mathrm{~m}^{2} / \mathrm{s}^{2}$ in linear drift occurs every day based on the precise scale and bias factors in Table 1 . Therefore, the scale and bias factors of the non-conservative force data from the space-borne accelerometers derived using the EGM2008 priori Earth gravitational field model are effective. At the same time, we calibrated the nonconservative force data based on the GGM02S and EIGEN-5C priori Earth's gravitational field models. As represented in Table 1, the scale and bias factors from the GGM02S and EIGEN-5C models are respectively close to those from the EGM2008 model. All in all, the calibration of the non-conservative force data from the space-borne accelerometers based on the energy conservation principle is feasible.

The physical explanation why the disturbing geopotential error represents linear drift is expressed as follows: The accuracy of the non-conservative force data is higher only within the frequency range from $10^{-4}$ to $10^{-1} \mathrm{~Hz}$; however, the systematic error increases outside this frequency band. Because the dissipative energy difference,

$$
\boldsymbol{E}_{\mathrm{f} 12}=\int\left(\dot{\boldsymbol{r}}_{2} \cdot \boldsymbol{f}_{2}-\dot{\boldsymbol{r}}_{1} \cdot \boldsymbol{f}_{1}\right) \mathrm{d} t
$$

represents as integral form, there is a drift in the energy difference of Eq. (6). The gravitational signal is sensitive to the

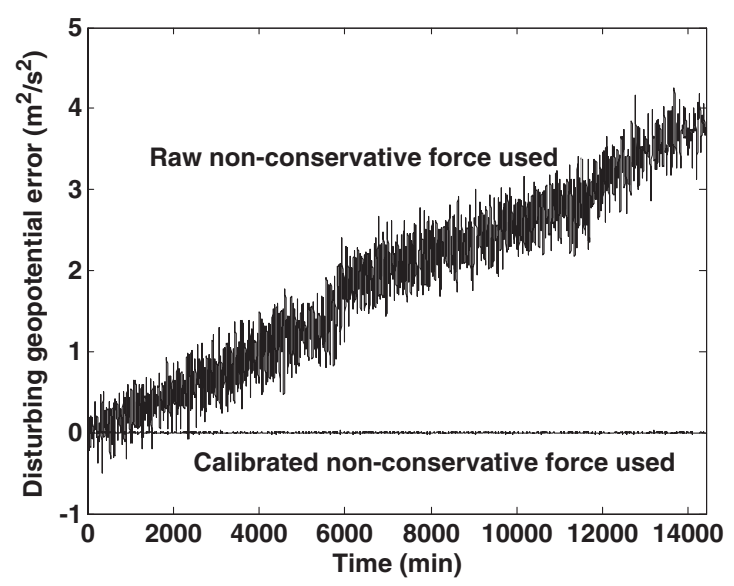

Fig. 2. Errors of disturbing geopotential from the twin GRACE satellites.

systematic error in the non-conservative force data. Accordingly, precise calibration is crucial to highly accurate recovery of the Earth's gravitational field with high spatial resolution.

\section{Conclusions}

(1) In the GRACE-Level-1B data, the real accuracy $\left(f_{x}=3 \times 10^{-9} \mathrm{~m} / \mathrm{s}^{2}, f_{y, z}=3 \times 10^{-10} \mathrm{~m} / \mathrm{s}^{2}\right)$ of non-conservative force data from the space-borne accelerometers basically matches the anticipated accuracy $\left(f_{x}=\right.$ $\left.10^{-9} \mathrm{~m} / \mathrm{s}^{2}, f_{y, z}=10^{-10} \mathrm{~m} / \mathrm{s}^{2}\right)$, and the real accuracy $(5 \times$ $10^{-5} \mathrm{rad}$ ) of attitude data from star camera assembly is, on the whole, true to the anticipated accuracy $\left(3 \times 10^{-5} \mathrm{rad}\right)$.

(2) Different from former calibration using the dynamic method, we newly calibrated the GRACE-Level-1B nonconservative force data between 2009-01-01-12-31, as released by the JPL, using the EGM2008, GGM02S and EIGEN-5C priori Earth gravitational field models based on an improved energy conservation principle, and provide a reasonable physical explanation. The results show that there is a linear drift of about $0.4 \mathrm{~m}^{2} / \mathrm{s}^{2}$ each day in the disturbing geopotential error due to systematic error in the raw non-conservative force data. However, error is only $0.01 \mathrm{~m}^{2} / \mathrm{s}^{2}$ after effective calibration. The advantages of calibration using the energy conservation principle are as follows:

(a) The physical meaning is definite. The energy conservation principle is one of the widely used laws in physics. Its characteristics are that the Earth's gravitational field 
can be recovered easily because the observation equation has a linear relationship between geopotential coefficients and the disturbing geopotential of Earth's gravitational field.

(b) The requirements for computer performance are low. The non-conservative force data from the space-borne accelerometers are efficiently calibrated and the Earth's gravitational field can be rapidly recovered using a personal computer (CPU of $3.0 \mathrm{GHz}$ and Memory of $4 \mathrm{~Gb}$ in this study) applying the energy conservation principle. However, this is generally calibrated using a mainframe parallel computer applying the dynamic method.

(c) The computational complexity is reduced while guaranteeing calibration accuracy. Based on the energy conservation principle, the calibration of non-conservative force data from the space-borne accelerometers and the recovery of the Earth's gravitational field complete up to degree and order 120 will consume $6 \mathrm{~h}$ using the observation parameters (duration of $30 \mathrm{~d}$ and sample intervals of $5 \mathrm{~s}$ ) and a personal computer (CPU of $3.0 \mathrm{GHz}$ and memory of $4 \mathrm{~Gb}$ ). The computing time using the dynamic method is at lease five times longer than when using the energy conservation principle with the same observation parameters and personal computer.

(3) The Earth's gravitational field signal is sensitive to the systematic error of non-conservative force data from the space-borne accelerometers. Accordingly, precise scale and bias factors are important for guaranteeing accurate recovery of the Earth's gravitational field with high spatial resolution.

\section{Acknowledgments}

We greatly appreciate the helpful suggestions from editor, anonymous referees, and Prof. Jun Luo, School of Physics, Huazhong University of Science and Technology, China. This work was supported by the Main Direction Program of Knowledge Innovation of the Chinese Academy of Sciences for Distinguished Young Scholars (Grant No. KZCX2-EW-QN114), the National Natural Science Foundation of China for Young Scholars (Grant No. 41004006), the Natural Science Foundation of Hubei Province of China (Grant No. 2010CDB05301), the Open Research Foundation of the Key Laboratory of Geo-Informatics of the State Bureau of Surveying and Mapping (Grant No. 201031), the Open Research Foundation of the Key Laboratory of Dynamic Geodesy of the Chinese Academy of Sciences (Grant No. L09-14), the Frontier Field Program of Knowledge Innovation of the Institute of Geodesy and Geophysics of the Chinese Academy of Sciences, the Hubei Province Key Laboratory of Refractories and Ceramics Ministry-Province Jointly-Constructed Cultivation Base for the State Key Laboratory (Grant No. G201009), and the Open Research Fund Program of Hubei Province Key Laboratory of Systems Science in Metallurgical Process (Grant No. C201019).

\section{References}

1) O'Keefe, J. A.: An Application of Jacobi's Integral to the Motion of an Earth Satellite, Astron. J., 62 (1957), pp. 265-266.

2) Visser, P. N. A. M.: Gravity Field Determination with GOCE and GRACE, Adv. Space Res., 23 (1999), pp. 771-776.

3) Balmino, G.: Gravity Field Recovery from GRACE: Unique Aspects of the High Precision Inter-Satellite Data and Analysis Methods, Space Sci. Rev., 108 (2003), pp. 47-54.

4) Tapley, B. D., Bettadpur, S., Ries, J. C., Thompson, P. F. and Watkins, M. M.: GRACE Measurements of Mass Variability in the Earth System, Science, 305 (2004), pp. 503-505.

5) Yamamoto, K., Otsubo T., Kubo-oka, T. and Fukuda, Y.: A Simulation Study of Effects of GRACE Orbit Decay on the Gravity Field Recovery, Earth Planets Space, 57 (2005), pp. 291-295.

6) Chen, J. L., Wilson, C. R. and Tapley, B. D.: Satellite Gravity Measurements Confirm Accelerated Melting of the Greenland Ice Sheet, Science, 313 (2006), pp. 1958-1960.

7) Han, S. C., Shum, C. K., Bevis, M., Ji, C. and Kuo, C. Y.: Crustal Dilatation Observed by GRACE after the 2004 Sumatra-Andaman Earthquake, Science, 313 (2006), pp. 658-662.

8) Hosokawa, S., Masumoto, Y., Takezawa, S. and Haneji, K.: Damping Control by One-Axis Magnetic Torquer and Gravity Gradient Attitude Stabilization for Small Satellite, Trans. Jpn. Soc. Aeronaut. Space Sci., 49 (2006), pp. 94-100.

9) Xu, P. L.: Position and Velocity Perturbations for the Determination of Geopotential from Space Geodetic Measurements, Celest. Mech. Dyn. Astron., 100 (2008), pp. 231-249.

10) Zheng, W., Shao, C. G., Luo, J. and Xu, H. Z.: Improving the Accuracy of GRACE Earth's Gravitational Field using the Combination of Different Inclinations, Prog. Nat. Sci., 18 (2008), pp. 555-561.

11) Zheng, W., Xu, H. Z., Zhong, M. and Yun, M. J.: Accurate and Rapid Error Estimation on Global Gravitational Field from Current GRACE and Future GRACE Follow-On Missions, Chin. Phys. B, 18 (2009), pp. 3597-3604.

12) Zheng, W., Xu, H. Z., Zhong, M., Yun, M. J., Zhou, X. H. and Peng, B. B.: Effective Processing of Measured Data from GRACE Key Payloads and Accurate Determination of Earth's Gravitational Field, Chin. J. Geophys., 52 (2009), pp. 772-782.

13) Zheng, W., Xu, H. Z., Zhong, M. and Yun, M. J.: An Analysis on Requirements of Orbital Parameters in Satellite-to-Satellite Tracking Mode, Chinese Astron. Astr., 34 (2010), pp. 413-423.

14) Zheng, W., Xu, H. Z., Zhong, M. and Yun, M. J.: Demonstration of Requirement on Future Lunar Satellite Gravity Exploration Mission based on Interferometric Laser Intersatellite Ranging Principle, J. Astronautics, 32 (2011), pp. 922-932.

15) Reigber, Ch., Schmidt, R. and Flechtner, F.: An Earth Gravity Field Model Complete to Degree and Order 150 from GRACE: EIGEN-GRACE02S, J. Geodyn., 39 (2005), pp. 1-10.

16) Tapley, B., Ries, J., Bettadpur, S., Chambers, D., Cheng, M., Condi, F., Gunter, B., Kang, Z., Nagel, P., Pastor, R., Pekker, T., Poole, S. and Wang, F.: GGM02-An Improved Earth Gravity Field Model from GRACE, J. Geod., 79 (2005), pp. 467-478.

17) Jekeli, C.: The Determination of Gravitational Potential Differences from SST Tracking, Celest. Mech. Dyn. Astron., 75 (1999), pp. 85101.

18) Han, S. C.: Efficient Determination of Global Gravity Field from Satellite-to-Satellite Tracking Mission, Celest. Mech. Dyn. Astron., 88 (2004), pp. 69-102. 